\section{Atrioventricular nodal ablation and implantation of mode switching dual chamber pacemakers: effective treatment for drug refractory paroxysmal atrial fibrillation}

\author{
H J Marshall, Z I Harris, M J Griffith, M D Gammage
}

Department of

Cardiovascular Medicine, University of Birmingham, Queen Elizabeth Hospital, Edgbaston,

Birmingham B15 2TH, UK

H J Marshall

Z I Harris

M J Griffith

M D Gammage

Correspondence to: Dr Marshall.

Accepted for publication 31 December 1997

\begin{abstract}
Objective-To assess the effect of atrioventricular node ablation and implantation of a dual chamber, mode switching pacemaker on quality of life, exercise capacity, and left ventricular systolic function in patients with drug refractory paroxysmal atrial fibrillation.

Patients-18 consecutive patients with drug refractory paroxysmal atrial fibrillation.

Methods-Quality of life was assessed before and after the procedure using the psychological general wellbeing index (PGWB), the McMaster health index (MHI), and a visual analogue scale for cardiac symptoms. Nine of the patients also underwent symptom limited exercise tests and echocardiography to assess left ventricular systolic function.

Results-The procedure allowed a reduction in antiarrhythmic drug treatment $(p<0.01)$. PGWB and symptom scores improved $(p<0.01)$ but the MHI score did not change. Left ventricular systolic function and exercise capacity were unchanged.

Conclusions-Atrioventricular node ablation and implantation of a DDDR/MS pacemaker is effective treatment for refractory paroxysmal atrial fibrillation, producing improved quality of life while allowing a reduction in drug burden. The popularity of the treatment is justified, but further studies are needed to determine optimum timing of intervention. (Heart 1998;79:543-547)
\end{abstract}

Keywords: paroxysmal atrial fibrillation; drug refractoriness; atrioventricular node ablation; dual chamber pacemaker

Atrial fibrillation is the most common sustained cardiac arrhythmia, with an overall prevalence of $0.5-1 \%$ in the USA. ${ }^{1}$ The prevalence increases with age and it is often paroxysmal for some time before developing into the permanent form. ${ }^{2}$ In many patients, symptoms such as palpitations, breathlessness, and chest pain can be disabling. These can often be controlled by drugs but in many patients atrial fibrillation still occurs despite multiple trials of antiarrhythmic drug treatment. In addition, the drugs used to prevent paroxysmal atrial fibrillation (for example, sotalol, flecainide, propafenone, amiodarone) have side effect profiles which are either poorly tolerated by some patients or preclude their use because of coexistent medical conditions such as ischaemic heart disease, impaired left ventricular function, or asthma. This means that there are many patients in whom an alternative mode of treatment is desirable.

Since the early 1980s, atrioventricular node ablation has been possible using transvenous techniques. ${ }^{3}$ With the development of radiofrequency ablation this has become a safe, easily performed procedure. ${ }^{4}$ Perhaps motivated by this, permanent pacemaker technology has advanced over the last decade so that there are now many dual chamber, mode switching devices available, each using a different algorithm to allow atrioventricular synchronous pacing during sinus rhythm but ventricular (VVI) or ventricular rate responsive pacing (VVIR) during atrial tachyarrhythmias. ${ }^{5-8} \mathrm{Be}-$ cause atrial sensing continues during tachycardia, the modes switched to are actually DDI or DDIR; atrial monitoring is of course required to enable the device to switch back to DDD mode on the resumption of sinus rhythm. As a result, atrioventricular node ablation and dual chamber mode switching (DDDR/MS) pacing is becoming accepted as treatment for drug refractory paroxysmal atrial fibrillation. At present, however, there is little available evidence to support this practice. Although several studies have shown an improvement in quality of life ${ }^{9-11}$ and left ventricular systolic function, ${ }^{12-14}$ the data come either from mixed populations of permanent and paroxysmal fibrillators $^{10}{ }^{12-14}$ or from patients who have received VVIR or non-mode switching pacemakers. ${ }^{911}$ No study to date has specifically assessed the effects of atrioventricular node ablation combined with the more complex technology of DDDR/MS pacing in a group of patients with drug refractory paroxysmal atrial fibrillation.

The aim of this study was to assess the effects of atrioventricular node ablation and DDDR/MS pacing on quality of life, exercise capacity, and left ventricular systolic function in a group of patients with drug refractory paroxysmal atrial fibrillation.

\section{Methods}

STUDY SETTING AND POPULATION

The study was set in a tertiary referral centre for cardiac electrophysiology. We studied 18 consecutive patients (12 male, six female) who 
Table 1 Quality of life scores, antiarrhythmic drug burden, left ventricular fractional shortening, and exercise capacity before and after atrioventricular node ablation and implantation of a dual chamber mode switching pacemaker

\begin{tabular}{lllllll}
\hline & $\begin{array}{l}\text { PGWB (out of } \\
110)\end{array}$ & $\begin{array}{l}\text { MHI (out of } \\
\text { 20) }\end{array}$ & $\begin{array}{l}\text { Symptom } \\
\text { score }\end{array}$ & $\begin{array}{l}\text { Number of } \\
\text { drugs }\end{array}$ & FS\% & $\begin{array}{l}\text { Exercise } \\
\text { time (s) }\end{array}$ \\
\hline $\begin{array}{l}\text { Before } \\
\text { After }\end{array}$ & $\begin{array}{l}79.44 \\
\text { p value }\end{array}$ & 14.11 & 50.44 & 1.38 & $34.47(1) \dagger$ & $600(0)$ \\
\hline 0.01 & 15.22 & 21.56 & 0.06 & $31.65(3)$ & $586(3)$ \\
\hline
\end{tabular}

PGWB, psychological general wellbeing score; MHI, McMaster health index score.

*Antiarrhythmic drugs; †number of patients in atrial fibrillation at time of testing.

were referred for atrioventricular node ablation and DDDR/MS pacing for drug refractory paroxysmal atrial fibrillation. Mean age at ablation was 63 years (range 39 to 76 ). Only two patients had any significant non-cardiac medical history, both having quite limiting obstructive airways disease. The aetiology of atrial fibrillation was as follows: two had mitral valve disease (one with mitral valve replacement); three had ischaemic heart disease (not suitable for revascularisation); 13 had lone atrial fibrillation. Patients had tried a mean (SD) of 5.3 (3.0) different drugs or drug combinations before referral for atrioventricular node ablation.

\section{PROCEDURES}

We obtained informed consent for the procedure from all patients. Radiofrequency ablation of the atrioventricular node was performed under local anaesthesia and intravenous sedation with diazepam and diamorphine where required. A $7 \mathrm{~F}$ Polaris thermistor catheter (Cordis, Baldwin Park, California, USA) was passed from the right femoral vein, with a temporary pacing wire in the right ventricle; the compact atrioventricular node was located using the electrogram described by Scheinman et $a l,{ }^{3}$ and energy was applied to achieve a temperature of $70^{\circ} \mathrm{C}$ for 60 seconds. The catheter was repositioned and energy reapplied if atrioventricular block was not achieved with each burn. After the induction of third degree atrioventricular block, a dual chamber, mode switching pacemaker was implanted through the left subclavian vein (Medtronic Thera DR $7940, \mathrm{n}=12$ (Medtronic, Minneapolis, Minnesota, USA); Vitatron Diamond, $n=5$ (Vitatron, Dieren, The Netherlands); CPI Vigor $\mathrm{DR}, \mathrm{n}=1$ (CPI, St Paul, Minnesota, USA)).

Before and six weeks after intervention, we assessed the patients' quality of life using the psychological general wellbeing questionnaire (PGWB), ${ }^{15}$ the McMaster health index questionnaire $(\mathrm{MHI})^{16}$ (physical component), and a cardiac symptom score (CSS). ${ }^{17}$ The PGWB is a 22 part multichoice questionnaire which estimates patients' perception of their emotional status, general health, and response to illness; it generates an overall wellbeing score out of 110 , with a higher score representing a perception of better health. The MHI is a 20 part questionnaire requiring yes/no answers and assesses patients' ability to perform activities of daily living. It is scored out of 20, with a higher score indicating greater functional ability. The CSS is a visual analogue scale questionnaire in 11 parts assessing cardiac symptoms (chest pain, breathlessness, palpitation, dizziness), rating

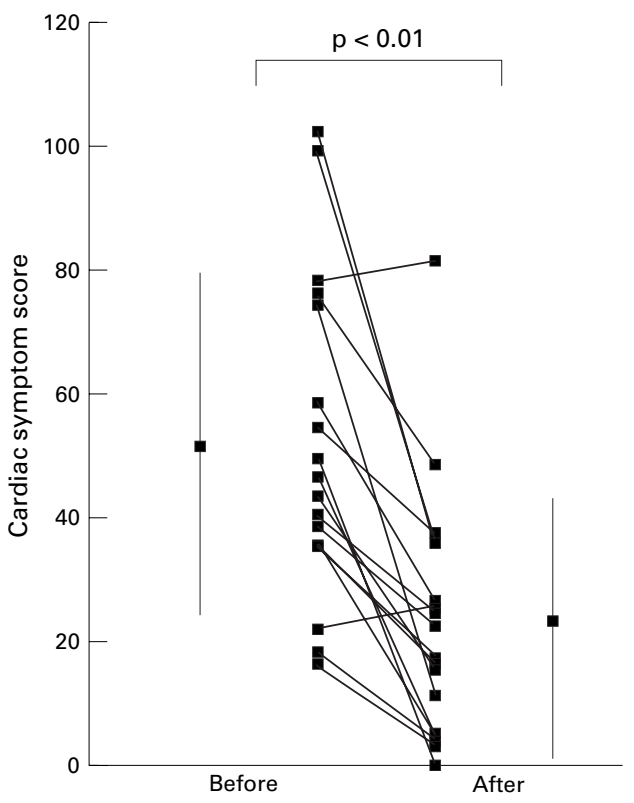

Figure 1 Individual cardiac symptom scores before and six weeks after atrioventricular node ablation. Error bars show the mean score and standard deviation.

each response on a scale from 0 to 10 . The final score is out of 110 , with a lower score indicating a lower level of symptoms. We chose these tools as they are all well validated ${ }^{15-17}$ and have been used for the evaluation of pacemaker patients. ${ }^{9}{ }^{17}$ In addition, each assesses a different aspect of quality of life.

Nine patients also underwent symptom limited exercise testing using the chronotropic assessment exercise protocol (CAEP) ${ }^{18}$ and echocardiography to assess left ventricular systolic function (fractional shortening). ${ }^{19}$

\section{STATISTICAL METHODS}

Quality of life scores before and after intervention were compared using Wilcoxon's signed rank test (normative data for these tools is non-parametric), and exercise capacity and left ventricular fractional shortening changes were assessed using a paired Student's $t$ test.

\section{PACEMAKER PROGRAMMING}

All pacemakers were programmed to DDDR mode with mode switching "on" for the Vigor and Thera patients and "Auto" for the diamond patients. Sensing was bipolar in the atrium (to reduce the risk of myopotential and far field $\mathrm{R}$ wave sensing) and atrial sensitivity programmed to $0.5 \mathrm{mV}$ to maximise accurate sensing during atrial fibrillation. Ventriculoatrial intervals were checked (where possible with the pacemaker diagnostics) with atrial blanking at nominal settings to exclude far field $R$ wave sensing and atrial blanking prolonged if this was detected. Routine pacemaker checks were carried out six weeks after implantation.

\section{Results}

Third degree atrioventricular block was easily achieved in all patients with no complications, and was maintained at follow up. The mean number of burns required to achieve complete 


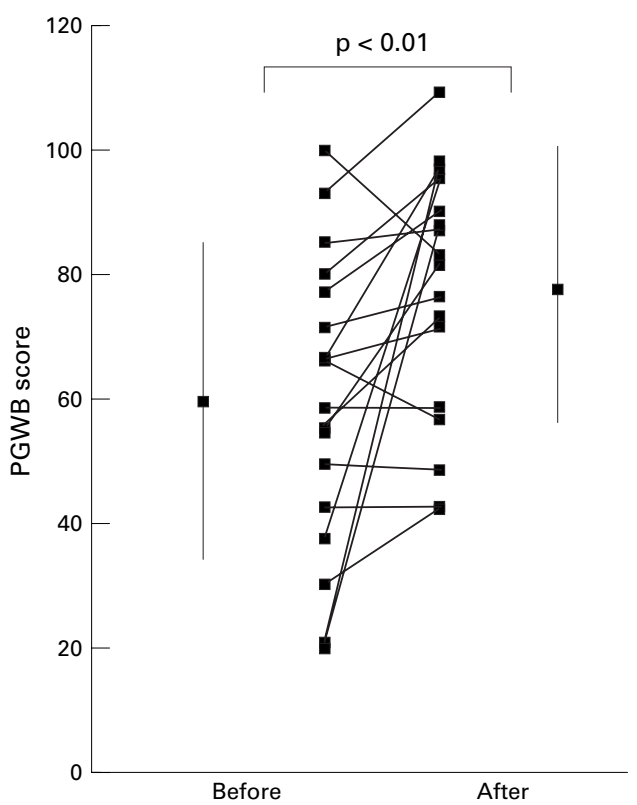

Figure 2 Individual psychological general wellbeing sores before and six weeks after atrioventricular node ablation. Error bars show the mean score and standard deviation.

heart block was 2.6 (range 1 to 12 ) and it was not necessary to proceed to a combined left and right sided approach in any patient. Pacemaker function was satisfactory in all patients both immediately and at six week follow up. In particular, all patients had satisfactory atrial sensing. Pacemaker interrogation at this stage suggested that four patients $(22 \%)$ had developed persistent atrial fibrillation; patients were left programmed to DDDR/MS in case sinus rhythm returned.

Table 1 summarises the quality of life scores, antiarrhythmic drug burden, left ventricular fractional shortening, and exercise capacity before and after intervention. It can be seen that we were able to reduce the number of antiarrhythmic drugs taken following the procedure $(p<0.01)$ and that there was an improvement in the psychological general wellbeing $(\mathrm{p}<0.01$; fig 1$)$ and cardiac symptom scores $(p<0.01$; fig 2$)$. Before the intervention, all patients had normal left ventricular systolic function (FS $>30 \%$ ). We were unable to show any significant change in left ventricular fractional shortening, exercise tolerance, or the MHI score after intervention.

\section{Discussion}

Previous studies of atrioventricular node ablation and pacemaker implantation have demonstrated its feasibility ${ }^{4}$ and an improvement in quality of life $\mathrm{e}^{9120}$ with its use in certain populations. However, these studies have not specifically addressed the use of dual chamber, mode switching technology in combination with atrioventricular node ablation for patients with paroxysmal atrial fibrillation. A criticism of some of the studies is that they have applied the technique to mixed populations of chronic and paroxysmal fibrillators ${ }^{10}$ or even to other supraventricular arrhythmias, ${ }^{14}$ thus making it difficult to apply the findings specifically to patients with either chronic or paroxysmal atrial fibrillation. Kay's original series ${ }^{9}$ did focus on paroxysmal fibrillators but this was before the availability of radiofrequency ablation and only VVIR pacing was used. It does not, therefore, directly support the use of DDDR/MS pacemakers in this population. The only other study which looked specifically at paroxysmal fibrillators did use dual chamber pacing $^{11}$; however, this was a retrospective survey of 36 patients and the devices implanted did not incorporate mode switching technology.

Despite this lack of evidence, current pacing prescription guidelines ${ }^{21} 22$ advocate the use of DDDR/MS in this context, and prescription patterns would suggest that these guidelines are becoming widely accepted. Our study, however, shows that atrioventricular node ablation and implantation of a dual chamber mode switching pacemaker is an effective treatment for drug refractory paroxysmal atrial fibrillation. In the population studied it allowed a significant reduction in antiarrhythmic drug burden while producing an improvement in quality of life and cardiac symptoms. These improvements were detected by the psychological general wellbeing index and the cardiac symptom score. The MHI did not improve significantly; we suggest that this was because it assesses patients' general health in terms of everyday functional ability, and our patientsalthough highly symptomatic and psychologically affected by their symptoms-were not very limited functionally. This may be exaggerated by the fact the MHI only asks patients to assess their functional ability on the day of assessment and hence the preoperative score may already be high if the patient is assessed in sinus rhythm, as all of ours were. Indeed, several of the patients attained a maximum score before intervention, thus allowing no room for improvement. This finding is in keeping with those of Fitzpatrick et al, ${ }^{11}$ whose retrospective assessment was able to show an improvement in quality of life and cardiac symptoms but not in the activities of daily living in paroxysmal fibrillators (though there was a significant improvement in all three in chronic fibrillators).

Our patients' pacemaker prescription was dictated by physician preference and hence the mode switching algorithms employed varied between patients. The CPI Vigor DR and the Medtronic Thera DR both have counter based algorithms which require a certain number of cardiac cycles to detect atrial fibrillation and hence may take 5-10 seconds to mode switch; in contrast the Vitatron Diamond device has a "beat to beat" algorithm which will switch on the first beat of atrial fibrillation (or on a single atrial extrasystole). The advantage of the latter algorithm is that a more constant heart rate is maintained at the onset of atrial fibrillation rather than the pacemaker tracking to the upper rate limit and only falling back to the sensor rate once the arrhythmia is diagnosed. Despite the fact that the majority of our patients had slow mode switching devices, we were still able to demonstrate the improvements in quality of life described above. It is 
possible that the improvements may have been greater if all patients had received a device with a "faster" algorithm. ${ }^{23}$

In any study of the impact of a procedure on quality of life and wellbeing, a placebo effect must be considered. None of the studies of atrioventricular node ablation and pacing for paroxysmal atrial fibrillation (including ours) assesses this problem. However, Brignole et al have addressed this in a short term study of a mixed group of patients with chronic atrial fibrillation and flutter. ${ }^{20}$ They randomised patients to undergo either pacemaker implantation alone or atrioventricular node ablation, and VVIR pacing at the same session. Patients were assessed before and 15 days after their procedure, using a symptom score, New York Heart Association classification, and an activity scale. While there were improvements in the group treated with pacing alone, the improvement in the ablated patients was nearly twice as great, suggesting that although there may be some placebo effect associated with undergoing a procedure, this does not account for all the benefits observed. It might also be argued that even if there is a placebo effect associated with the procedure, it is still beneficial if patients' perception of wellbeing is improved.

In contrast to the effects on quality of life, we were unable to show any change in exercise capacity after the procedure. Only Kay's study ${ }^{9}$ has looked at this in paroxysmal fibrillators, when an improvement was seen. Brignole et al found a similar effect in chronic fibrillators, as might be expected..$^{20}$ We suggest that, as for the MHI scores, we did not detect a change in exercise capacity because the functional status of our patients was generally good before intervention. This difference from Kay's population ${ }^{9}$ may reflect the fact that atrioventricular node ablation is now relatively commonplace and that at the time of Kay's study it would have been considered much more a treatment of "last resort." The lack of improvement in exercise capacity in our population may also be related to the fact that all our patients were exercised in sinus rhythm at baseline, but three were in atrial fibrillation at follow up; hence there would have been a loss of atrial transport in these patients at the time of their postoperative test. It is important to note, however, that despite these factors we did not find a deterioration in exercise capacity after intervention.

With regard to the effects of atrioventricular node ablation and pacing for atrial fibrillation on left ventricular systolic function, published reports to date contain no studies using the DDDR/MS pacing mode and, as with previous quality of life studies, the populations studied have been heterogeneous with regard to the chronic or paroxysmal nature of the arrhythmia. $^{12} 1324$ However, in patients with impaired left ventricular function and chronic atrial fibrillation, atrioventricular node ablation and pacing seems to improve left ventricular systolic function. ${ }^{20}{ }^{25}$ It is suggested that in paroxysmal atrial fibrillation the degree of improvement is related to the frequency and duration of atrial fibrillation and is again restricted to those patients with marked left ventricular impairment. ${ }^{13}$ In our study there was no beneficial effect on left ventricular systolic function. The lack of improvement is perhaps not surprising as most of the patients were assessed in sinus rhythm and all had normal left ventricular function before ablation. It is important to note, however, that as with studies of patients with chronic atrial fibrillation and normal left ventricular function, we did not show any detrimental effect of the procedure on left ventricular fractional shortening, despite the potentially negative inotropic effect of right ventricular apical pacing. ${ }^{26}$

The results of this study included four patients $(22 \%)$ in whom atrial fibrillation was paroxysmal at the time of ablation and pacing, but who rapidly progressed to persistent atrial fibrillation on withdrawal of antiarrhythmic drugs. All of these patients had an improvement in their quality of life scores after intervention. It seems likely that they would have fared equally well with VVIR pacing; however, as their atrial fibrillation was clearly paroxysmal at the time of ablation and their pacemakers were left programmed to DDDR/MS on an intention to treat basis, we feel their inclusion in the results was justified. It is evident, however, that further work is required to identify risk factors for progression to chronic atrial fibrillation in a population of patients undergoing ablation and pacing for paroxysmal atrial fibrillation.

In conclusion, we found that atrioventricular node ablation and implantation of a DDDR/MS pacemaker was an effective treatment for paroxysmal atrial fibrillation. We were unable to show any detrimental effect on left ventricular systolic function or exercise capacity. However, it remains unclear whether DDDR/MS pacing is superior to VVIR, or indeed whether optimising medical treatment may achieve the same results.

HJM and ZIH are supported by the British Heart Foundation.

1 Kannel WB, Abbott RD, Savage DD, et al. Epidemiologic features of atrial fibrillation; the Framingham study. $N$ Engl Med 1982;306:1018-22.

2 Murgatroyd FD, Camm AJ. Atrial arrhythmias. Lancet 1993;341:1317-22.

3 Scheinman MM, Morady F, Hess DS, et al. Catheter-induced ablation of the atrioventricular junction to control refractory supraventricular arrhythmias. $\mathcal{F} A M A$ 1982;248:851-5.

4 Yeung-Lai-Wah J, Alison J, Lonergan L, et al. High success rate of atrioventricular node ablation with radiofrequency energy. I Am Coll Cardiol 1991;18:1753-8.

5 Levine PA, Bornzin GA, Barlow J, et al. A new auto mode witch algorithm for supraventricular arrhythmias. PACE 1994;17:1895-9.

6 Den Dulk K, Dijkman B, Pieterse M, et al. Initial experience with mode switching in a dual sensor, dual chamber pacemaker in patients with paroxysmal atrial tachyarrhythmias. maker in patients with $1994 ; 17: 1900-7$.

7 Ovsyshcher IE, Katz A, Bondy C. Initial experience with a new algorithm for automatic mode switching from DDDR to DDIR mode. PACE 1994;17:1908-12.

8 Provenier F, Jordans L, Verstraeten T, et al. The "automatic mode switch" function in successive generations of minute ventilation sensing dual chamber rate responsive pacemakers. PACE 1994;17:1913-19.

9 Kay GN, Bubien RS, Epstein AE, et al. Effect of catheter ablation of the atrioventricular junction on quality of life and exercise tolerance in paroxysmal atrial fibrillation. $\mathrm{Am}$ f Cardiol 1988;62:741-4.

10 Mennozzi C, Brignole M, Gianfranchi L, et al. Radiofrequency catheter ablation and modulation of the atrioventricular node in patients with atrial fibrillation. PACE 1994; 17:2143-9.

11 Fitzpatrick AP, Kourouyan HD, Siu A, et al. Quality of life and outcomes after radiofrequency His-bundle catheter ablation and permanent pacemaker implantation: impact of treatment in paroxysmal and established atrial fibrillaof treatment in paroxysmal and establit
tion. Am Heart f 1996;131:499-507. 
12 Edner M, Ciadahl K, Bergfeldt L, et al. Prospective study of left ventricular function after radiofrequency ablation of the atrioventricular junction in patients with atrial fibrillation. Br Heart f 1995;74:261-7.

13 Rodriguez LM, Smeets JLRM, Baiyan X, et al. Improvement in left ventricular function by ablation of atrioven tricular nodal conduction in selected patients with lone atrial fibrillation. Am f Cardiol 1993;72:1137-41.

14 Rosenquist M, Lee MA, Moulinier L, et al. Long term follow up of patients after trans catheter direct curren ablation of the atrioventricular node. $\mathcal{F} \mathrm{Am}$ Coll Cardiol 1990;16:1467-74.

15 Chambers LW, Depuy HJ. Assessment of quality of life in clinical trials of cardiovascular therapies. New York: Le Jacq Publishing, 1984:160-4.

16 Chambers LW, Depuy HJ. Assessment of quality of life in clinical trials of cardiovascular therapies. New York: Le Jacq Publishing, 1984:170-83.

17 Linde-Edelstam C, Nordlander R, Unden A, et al. Quality of life in patients treated with atrioventricular synchronous pacing compared to rate modulated ventricular pacing: a pacing compared to rate modulated ventricular pacing: a
long term, double blind, cross-over study. PACE 1992;15: long term,

18 Wilkof B, Corey J, Blackburn G. A mathematical model of the chronotropic response to exercise. F Electrophysiol 1989; 3:176-80.

19 Feigenbaum H. Echocardiographic evaluation of cardiac chambers. In: Feigenbaum H, ed. Echocardiography. 5th ed. Pennsylvania: Lea and Febiger, 1994:143.
20 Brignole M, Gianfranchi L, Mennozzi C. Influence of atrioventricular junction radiofrequency ablation in patients with chronic atrial fibrillation and flutter on quality of life and cardiac performance. Am $\mathcal{F}$ Cardiol 1994;74:242-6.

21 Working party of the British Pacing and Electrophysiology Group. Recommendations for pacemaker prescription for symptomatic bradycardia. Br Heart f 1991;66:185-91.

22 ACC/AHA Task Force Report. Guidelines for implantation of cardiac pacemakers and antiarrhythmia devices. $\mathcal{F} \mathrm{Am}$ of cardiac pacemakers and
Coll Cardiol 1991;18:1-13.

23 Kay GN, Hess M, Marshall HJ, et al. Effect of modeswitching algorithms on patient symptoms. PACE 1997;20: 1064.

24 Twidale N, Sutton K, Bartlett L, et al. Effects on cardiac performance of atrioventricular node catheter ablation using radiofrequency current for drug refractory atrial using radiofrequency current for dr
arrhythmias. PACE 1993;16:1275-84.

25 Heinz G, Siostrzonek P, Kreiner G, et al. Improvement in left ventricular systolic function after successful radiofrequency His bundle ablation for drug refractory, chronic atrial fibrillation and recurrent atrial flutter. Am f Cardiol 1992;69:489-92.

26 Prinzen FW, Cheriex EC, Delhaas T, et al. Asymmetric thickness of the left ventricular wall resulting from asynchronous electrical activation : a study in dogs and patients with left bundle branch block. Am Heart $\mathcal{f}$ 1995;130:1045-53. 